\title{
Characterization of thermal, optical and carrier transport properties of porous silicon using the photoacoustic technique
}

\begin{abstract}
In this work, the porous silicon layer was prepared by the electrochemical anodization etching process on n-type and p-type silicon wafers. The formation of the porous layer has been identified by photoluminescence and SEM measurements. The optical absorption,energy gap, carrier transport and thermal properties of n-type and p-type porous silicon layers were investigated by analyzing the experimental data from photoacoustic measurements. The values of thermal diffusivity, energy gap and carrier transport properties have been found to be porosity-dependent. The energy band gap of n-type and p-type porous silicon layers was higher than the energy band gap obtained for silicon substrate $(1.11 \mathrm{eV})$. In the range of porosity (50-76\%) of the studies, our results found that the optical band-gap energy of p-type porous silicon (1.80-2.00 eV) was higher than that of the n-type porous silicon layer $(1.70-1.86 \mathrm{eV})$. The thermal diffusivity value of the n-type porous layer was found to be higher than that of the p-type and both were observed to increase linearly with increasing layer porosity.
\end{abstract}

Keyword: Porous silicon, Photoacoustic, Energy gap, Thermal properties 